\title{
PARAMETERIZED STRUCTURE-PRESERVING TRANSFORMATIONS OF MATRIX POLYNOMIALS*
}

\author{
DANIEL T. KAWANO ${ }^{\dagger}$
}

\begin{abstract}
This paper examines the relationship between the companion forms of regular matrix polynomials with singular leading coefficients. When two such polynomials have the same underlying finite and infinite Jordan structures, it is shown that their companion forms are connected by a strict equivalence transformation that can be parameterized using the commutant of the companion forms' common Weierstrass canonical form. The process developed herein for generating such parameterized transformations is applied to the useful class of diagonalizable quadratic polynomials.
\end{abstract}

Key words. Matrix polynomials, Isospectral, Structure-preserving transformations, Diagonalization.

AMS subject classifications. 15A21, 15A22, 15B05.

1. Introduction. We consider $n \times n$ matrix polynomials with degree $\ell: P(\lambda)=\sum_{i=0}^{\ell} A_{i} \lambda^{i}$, where $A_{i} \in \mathbb{C}^{n \times n}$ and $A_{\ell} \neq 0$. We restrict our attention to polynomials that are regular, i.e., polynomials for which $\operatorname{det}(P(\lambda)) \not \equiv 0$. When the leading coefficient $A_{\ell}$ is singular, some of the eigenvalues of $P(\lambda)$ are infinite. The finite eigenvalues of $P(\lambda)$ are those $\lambda \in \mathbb{C}$ such that $\operatorname{det}(P(\lambda))=0$. The eigenvalue at infinity corresponds to the zero eigenvalue of the reverse polynomial $P_{\mathrm{rev}}(\lambda)=\sum_{i=0}^{\ell} A_{\ell-i} \lambda^{i}$; the multiplicities of the infinite eigenvalue of $P(\lambda)$ and the zero eigenvalue of $P_{\text {rev }}(\lambda)$ are identical. Associated with $P(\lambda)$ are the two Jordan matrices $J_{F}$ and $J_{\infty}$, which we will often refer to as the finite Jordan form and infinite Jordan form, respectively, of $P(\lambda) ; J_{F}$ contains Jordan blocks of the polynomial's finite eigenvalues, while $J_{\infty}$ comprises Jordan blocks of the reverse polynomial's zero eigenvalue.

An $\ell n \times \ell n$ matrix pencil $A \lambda-B$ is called a linearization of a matrix polynomial $P(\lambda)$ when there exist unimodular polynomials $E(\lambda)$ and $F(\lambda)$ (that is, their determinants are nonzero constants) such that $E(\lambda)(A \lambda-B) F(\lambda)=P(\lambda) \oplus I$, in which case $A \lambda-B$ has the same Jordan form as $P(\lambda)$ for the finite eigenvalues [8]. The pencil $A \lambda-B$ is said to be a strong linearization of $P(\lambda)$ when, in addition to being a linearization of $P(\lambda)$, its reversal is a linearization of $P_{\text {rev }}(\lambda)$, thereby also preserving the Jordan structure for the infinite eigenvalue of $P(\lambda)[6,10]$. If $A \lambda-B$ is a strong linearization of $P(\lambda)$ and its coefficients are partitioned into $n \times n$ blocks that are each $0, \pm I$, or $\pm A_{i}$, then we call this strong linearization a companion form of $P(\lambda)$.

We say two polynomials $P(\lambda)$ and $\widetilde{P}(\lambda)$ are isospectral when their finite and infinite Jordan structures are identical. Suppose $A \lambda-B$ is a companion form of $P(\lambda)$. We denote the same companion form of $\widetilde{P}(\lambda)$ by $\widetilde{A} \lambda-\widetilde{B}$; that is, to obtain $\widetilde{A} \lambda-\widetilde{B}$, we replace all matrix coefficients $A_{i}$ of $P(\lambda)$ in $A \lambda-B$ with the corresponding coefficients $\widetilde{A}_{i}$ of $\widetilde{P}(\lambda)$. If $P(\lambda)$ and $\widetilde{P}(\lambda)$ are isospectral, then their companion forms are strictly equivalent, i.e.,

$$
U(A \lambda-B)=(\widetilde{A} \lambda-\widetilde{B}) V
$$

${ }^{*}$ Received by the editors on November 19, 2019. Accepted for publication on September 5, 2020. Handling Editor: Froilán Dopico.

${ }^{\dagger}$ Department of Mechanical Engineering, Rose-Hulman Institute of Technology, Terre Haute, Indiana 47803, USA (kawano@rose-hulman.edu). 
must hold for some invertible matrices $U, V \in \mathbb{C}^{\ell n \times \ell n}$. This type of transformation is called a structurepreserving transformation because it preserves the block structures of $A$ and $B$. The objective of our paper is to demonstrate that every nonsingular structure-preserving transformation $(U, V)$ relating a given pair of isospectral polynomials can be generated from a matrix $S$ that commutes with the common Weierstrass canonical form $\mathfrak{W}(\lambda)=\left(I \lambda-J_{F}\right) \oplus\left(J_{\infty} \lambda-I\right)=\left(I \oplus J_{\infty}\right) \lambda-\left(J_{F} \oplus I\right)$ of the polynomials' companion forms:

$$
\Phi:=\left\{S \in \mathbb{C}^{\ell n \times \ell n}: S \mathfrak{W}(\lambda)=\mathfrak{W}(\lambda) S\right\} .
$$

We first show that a parameterized structure-preserving transformation is possible for regular matrix polynomials over $\mathbb{C}$ and then specialize this result to polynomials with real coefficients. Because of their utility in applications, we also examine parameterized structure-preserving transformations relating quadratic polynomials to their diagonal forms (when diagonalization is possible).

Our findings herein are an extension of recent work in the area of parameterizing structure-preserving transformations. Lancaster and Zaballa [11] considered the case when the leading coefficient $A_{\ell}$ is invertible, i.e., when the eigenvalues of a matrix polynomial $P(\lambda)$ are all finite. They developed in [11] a method for characterizing structure-preserving transformations in terms of the centralizer $S$ of the (finite) Jordan form $J$ shared by the isospectral polynomials $P(\lambda)$ and $\widetilde{P}(\lambda): S J=J S$. Note that this is the same as $S$ commuting with the common Weierstrass canonical form of the polynomials' companion forms because $\mathfrak{W}(\lambda)=I \lambda-J_{F}=I \lambda-J$ in this case. Our goal is to establish the equivalent result for the more general case when $A_{\ell}$ is singular and to illustrate its application to diagonalizable quadratic polynomials. To strengthen the connection between Lancaster and Zaballa's work and ours, we follow a similar development and adopt similar notation and language. We should point out that a noticeable difference between our work and [11] is that Lancaster and Zaballa utilized a block-symmetric pencil

$$
L(\lambda)=\left[\begin{array}{ccccc}
A_{1} & A_{2} & \cdots & A_{\ell-1} & A_{\ell} \\
A_{2} & A_{3} & \cdots & A_{\ell} & 0 \\
\vdots & \vdots & . & \vdots & \vdots \\
A_{\ell-1} & A_{\ell} & \cdots & 0 & 0 \\
A_{\ell} & 0 & \cdots & 0 & 0
\end{array}\right] \lambda-\left[\begin{array}{ccccc}
-A_{0} & 0 & 0 & \cdots & 0 \\
0 & A_{2} & A_{3} & \cdots & A_{\ell} \\
\vdots & \vdots & \vdots & . & \vdots \\
0 & A_{\ell-1} & A_{\ell} & \cdots & 0 \\
0 & A_{\ell} & 0 & \cdots & 0
\end{array}\right],
$$

which is indeed a linearization of $P(\lambda)$ when $A_{\ell}$ is invertible. However, for singular $A_{\ell}$, this block-symmetric pencil is not a linearization of $P(\lambda)$ (see [13], especially Table 1 and Table 2 therein), and thus, it is not an acceptable choice for the pencils $A \lambda-B$ and $\widetilde{A} \lambda-\widetilde{B}$ in (1.1). Moreover, our method of generating a parameterized structure-preserving transformation is independent of the companion form of choice, but we demonstrate its use with the strong linearizations that have come to be called the Fiedler companion pencils [3] and with the special case of the familiar first companion form. Note that when $A_{\ell}$ is invertible, the block-symmetric linearization $L(\lambda)$ is strictly equivalent to any other linearization of $P(\lambda)$, and so all results presented herein reduce to those in [11].

We begin in Section 2 with a brief introduction of the companion forms that will appear throughout our paper for illustrative purposes (Section 2.1), and we follow this with a discussion about how to construct a structure-preserving transformation that relates two isospectral matrix polynomials with complex coefficients (Section 2.2). We next parameterize this transformation in Section 2.3 and then wrap up Section 2 with a detailed example in Section 2.4. In Section 3, we restrict our attention to real matrix polynomials, discussing how the theory over $\mathbb{C}$ in Section 2 is modified to accommodate real coefficients and real arithmetic; we demonstrate the usage of this modified theory with an example in Section 3.1. In Section 4, we give 
Electronic Journal of Linear Algebra, ISSN 1081-3810

A publication of the International Linear Algebra Society

Volume 36, pp. 723-743, November 2020

special attention to quadratic polynomials, either complex (Section 4.1) or real (Section 4.2), that can be diagonalized and provide an accompanying example (Section 4.3). Our paper concludes in Section 5 with a brief summary of key results.

2. Complex matrix polynomials. We first consider the general case when the coefficients of two isospectral matrix polynomials $P(\lambda)$ and $\widetilde{P}(\lambda)$ are complex. We only require that the leading coefficients be nonzero and that the polynomials be regular. Before we begin our work on parameterizing the structurepreserving transformation defined by (1.1), we briefly discuss a family of companion forms that will later be featured in illustrative examples.

2.1. Fiedler companion pencils. In [1], Antoniou and Vologiannidis introduced a method for generating a family of companion forms of a regular polynomial $P(\lambda)$ based on work by Fiedler concerning companion matrices for scalar polynomials [4]. These companion forms, which were later termed the Fiedler companion pencils (or Fiedler pencils for short) in [3], are obtained via multiplication of common factors in different orders. Specifically, for an $n \times n$ polynomial with degree $\ell$, define the $\ell+1$ matrices $M_{i} \in \mathbb{C}^{\ell n \times \ell n}$ $(i=0,1, \ldots, \ell)$, or Fiedler factors, as follows:

$$
\begin{gathered}
M_{0}=\left[\begin{array}{cc}
I_{(\ell-1) n} & 0 \\
0 & -A_{0}
\end{array}\right], \quad M_{\ell}=\left[\begin{array}{cc}
A_{\ell} & 0 \\
0 & I_{(\ell-1) n}
\end{array}\right], \\
M_{k}=\left[\begin{array}{cccc}
I_{(\ell-k-1) n} & 0 & 0 & 0 \\
0 & -A_{k} & I & 0 \\
0 & I & 0 & 0 \\
0 & 0 & 0 & I_{(k-1) n}
\end{array}\right] \quad(k=1,2, \ldots, \ell-1),
\end{gathered}
$$

where $I_{\alpha}$ denotes the $\alpha \times \alpha$ identity matrix. For a permutation $\sigma=\left(k_{0}, k_{1}, \ldots, k_{\ell-1}\right)$ of the indices $(0,1, \ldots, \ell-1)$, the Fiedler pencil associated with $\sigma$ is given by

$$
C_{F_{\sigma}}(\lambda)=M_{\ell} \lambda-M_{k_{0}} M_{k_{1}} \cdots M_{k_{\ell-1}}
$$

Owing to their structure, the Fiedler factors satisfy the commutativity relations $M_{i} M_{j}=M_{j} M_{i}$ for $|i-j| \neq 1$, implying that some Fiedler pencils associated with different permutations are the same. The set of Fiedler pencils includes the familiar first companion form,

$$
C_{1}(\lambda)=\left[\begin{array}{ccccc}
A_{\ell} & 0 & \cdots & 0 & 0 \\
0 & I & \cdots & 0 & 0 \\
\vdots & \vdots & \ddots & \vdots & \vdots \\
0 & 0 & \cdots & I & 0 \\
0 & 0 & \cdots & 0 & I
\end{array}\right] \lambda-\left[\begin{array}{ccccc}
-A_{\ell-1} & -A_{\ell-2} & \cdots & -A_{1} & -A_{0} \\
I & 0 & \cdots & 0 & 0 \\
0 & I & \cdots & 0 & 0 \\
\vdots & \vdots & \ddots & \vdots & \vdots \\
0 & 0 & \cdots & I & 0
\end{array}\right],
$$

which arises from the permutation $\sigma=(\ell-1, \ell-2, \ldots, 0)\left[1\right.$, Lemma 2.1]: $C_{1}(\lambda)=M_{\ell} \lambda-M_{\ell-1} M_{\ell-2} \cdots M_{0}$. The first companion form is well known to be a strong linearization of $P(\lambda)$ [6, Proposition 1.1]. Antoniou and Vologiannidis showed that any Fiedler pencil (2.5) is strictly equivalent to the first companion form and thus a strong linearization of $P(\lambda)$ [1, Theorem 2.3]. Generalizations of the Fiedler pencils have been developed over the years (see $[2,3,15]$ ), but in the examples herein demonstrating our forthcoming parameterized structure-preserving transformation, we limit our attention to the companion forms defined by (2.3)-(2.5). 
2.2. Establishing the structure-preserving transformation. Before we go about parameterizing the structure-preserving transformation $(U, V)$ defined in (1.1), we should first address how to determine nonsingular $U$ and $V$ that satisfy (1.1), which is not obvious. To do so, we will rely on the general theory of regular matrix polynomials developed by Gohberg, Kaashoek, and Lancaster in [6]. In what follows, $\Gamma$ is a Cauchy contour (i.e., the positively oriented boundary of a bounded Cauchy domain in $\mathbb{C}$ ) with inner domain $\Delta_{+}$and outer domain $\Delta_{-}=\mathbb{C}_{\infty} \backslash\left(\Delta_{+} \cup \Gamma\right)$, where $0 \in \Delta_{+}, \mathbb{C}_{\infty}=\mathbb{C} \cup\{\infty\}$ is the extended complex plane, and $\infty \in \Delta_{-}$[6, pp. 793-794].

First, we recall some definitions from [6, p. 794]. A regular polynomial $P(\lambda)$ is called $\Gamma$-regular if it is invertible for every $\lambda \in \Gamma$. Note that when $P(\lambda)$ is $\Gamma$-regular, so is any strong linearization (and thus any companion form) of $P(\lambda)$. A $\Gamma$-decomposed pencil is a $\Gamma$-regular pencil with the partitioned form $\left(I \lambda-T_{1}\right) \oplus\left(T_{2} \lambda-I\right)=\left(I \oplus T_{2}\right) \lambda-\left(T_{1} \oplus I\right)$, where $T_{1} \in \mathbb{C}^{m \times m}$ and $T_{2} \in \mathbb{C}^{(\ell n-m) \times(\ell n-m)}$ for some $m \in\{0,1, \ldots, \ell n\}, I \lambda-T_{1}$ is invertible for every $\lambda \in \Delta_{-} \cup \Gamma$, and $T_{2} \lambda-I$ is nonsingular for each $\lambda \in \Delta_{+} \cup \Gamma$.

Next, because of its importance in establishing the main result in our paper, we restate (with some rewording) Theorem 5.1 from [6].

Theorem 2.1 (Theorem 5.1 of [6]). Let $A \lambda-B$ be an $\ell n \times \ell n$ matrix pencil that is $\Gamma$-regular. Then $A \lambda-B$ is strictly equivalent to a $\Gamma$-decomposed pencil:

$$
Y^{-1}(A \lambda-B) X Y=\left[\begin{array}{cc}
I & 0 \\
0 & T_{2}
\end{array}\right] \lambda-\left[\begin{array}{cc}
T_{1} & 0 \\
0 & I
\end{array}\right],
$$

where the invertible $\ell n \times \ell n$ matrix

$$
X=\frac{1}{2 \pi \mathrm{i}} \int_{\Gamma}\left(1-\xi^{-1}\right)(A \xi-B)^{-1} \mathrm{~d} \xi
$$

and the invertible matrix $Y=\left[\begin{array}{llll}y_{1} & y_{2} & \cdots & y_{\ell n}\end{array}\right] \in \mathbb{C}^{\ell n \times \ell n}$ comprises a basis $y_{i}(i=1,2, \ldots, m)$ for the image of the $\ell n \times \ell n$ matrix

$$
W=\frac{1}{2 \pi \mathrm{i}} \int_{\Gamma} A(A \xi-B)^{-1} \mathrm{~d} \xi
$$

and a basis $y_{m+j}(j=1,2, \ldots, \ell n-m)$ for the kernel of $W$.

We refer the reader to the proof of Theorem 5.1 in [6] for explanations of why $X$ and $Y$ are nonsingular. A useful corollary of Theorem 2.1 is as follows.

Lemma 2.2. Let $P(\lambda)$ be an $n \times n$ matrix polynomial of degree $\ell$ that is $\Gamma$-regular, has nonzero leading coefficient, and has finite Jordan form $J_{F} \in \mathbb{C}^{n_{F} \times n_{F}}$ and infinite Jordan form $J_{\infty} \in \mathbb{C}_{\infty}^{n_{\infty} \times n_{\infty}}$, where $n_{F}+n_{\infty}=\ell n$. Define the Cauchy contour $\Gamma$ such that its inner domain contains the finite eigenvalues of $P(\lambda)$. Let $A \lambda-B$ be a strong linearization of $P(\lambda)$. Then $A \lambda-B$ is strictly equivalent to its Weierstrass canonical form $\mathfrak{W}(\lambda)=\left(I \oplus J_{\infty}\right) \lambda-\left(J_{F} \oplus I\right)$ :

$$
Q^{-1}(A \lambda-B) R=\left[\begin{array}{cc}
I & 0 \\
0 & J_{\infty}
\end{array}\right] \lambda-\left[\begin{array}{cc}
J_{F} & 0 \\
0 & I
\end{array}\right]=\mathfrak{W}(\lambda)
$$

for the invertible $\ell n \times \ell n$ matrices

$$
Q=Y Z \quad \text { and } \quad R=X Y Z,
$$


Electronic Journal of Linear Algebra, ISSN 1081-3810

A publication of the International Linear Algebra Society

Volume 36, pp. 723-743, November 2020.

where the $\ell \times \times n$ invertible matrices $X$ and $Y$ are computed as in Theorem 2.1 and the invertible blockdiagonal matrix $Z=\left(Z_{1} \oplus Z_{2}\right) \in \mathbb{C}^{\ell n \times \ell n}$ is such that the matrices $Z_{1} \in \mathbb{C}^{n_{F} \times n_{F}}$ and $Z_{2} \in \mathbb{C}^{n_{\infty} \times n_{\infty}}$ satisfy $Z_{1}^{-1} T_{1} Z_{1}=J_{F}$ and $Z_{2}^{-1} T_{2} Z_{2}=J_{\infty}$, for which $T_{1}$ is the $n_{F} \times n_{F}$ block in the upper-left corner of $Y^{-1} B X Y \in \mathbb{C}^{\ell n \times \ell n}$ and $T_{2}$ is the $n_{\infty} \times n_{\infty}$ block in the lower-right corner of $Y^{-1} A X Y \in \mathbb{C}^{\ell n \times \ell n}$.

Proof. Any choice of strong linearization $A \lambda-B$ is $\Gamma$-regular because $P(\lambda)$ is, and so $A \lambda-B$ is strictly equivalent to a $\Gamma$-decomposed pencil, i.e., (2.7) holds. When the inner domain of $\Gamma$ includes the finite eigenvalues of $P(\lambda)$, the eigenvalues of $T_{1}$ are these eigenvalues and 0 is the only eigenvalue of $T_{2}$ (see [6, p. 798]). Thus, because $A \lambda-B$ and $\left(I \oplus T_{2}\right) \lambda-\left(T_{1} \oplus I\right)$ are strictly equivalent, $Z_{1}^{-1} T_{1} Z_{1}=J_{F}$ and $Z_{2}^{-1} T_{2} Z_{2}=J_{\infty}$ for some invertible $Z_{1}$ and $Z_{2}$, where we see from (2.7) that $T_{1}$ is the $n_{F} \times n_{F}$ block in the upper-left corner of $Y^{-1} B X Y$ and $T_{2}$ is the $n_{\infty} \times n_{\infty}$ block in the lower-right corner of $Y^{-1} A X Y$. As a result, by utilizing the invertible matrix $Z=Z_{1} \oplus Z_{2}$, we can rewrite (2.7) as

$$
Y^{-1}(A \lambda-B) X Y=Z\left(\left[\begin{array}{cc}
I & 0 \\
0 & J_{\infty}
\end{array}\right] \lambda-\left[\begin{array}{cc}
J_{F} & 0 \\
0 & I
\end{array}\right]\right) Z^{-1}
$$

from which (2.10) and (2.11) follow.

Consequently, it is now clear how we can compute a structure-preserving transformation relating two isospectral polynomials for any choice of companion form.

Proposition 2.3. Let $P(\lambda)$ and $\widetilde{P}(\lambda)$ be $n \times n$ matrix polynomials of degree $\ell$ that are isospectral and $\Gamma$-regular and have nonzero leading coefficients. Define the Cauchy contour $\Gamma$ such that its inner domain contains the finite eigenvalues of $P(\lambda)$. Let $A \lambda-B$ and $\widetilde{A} \lambda-\widetilde{B}$ be companion forms of $P(\lambda)$ and $\widetilde{P}(\lambda)$, respectively. Then $(U, V)=\left(\widetilde{Q} Q^{-1}, \widetilde{R} R^{-1}\right)$ is a structure-preserving transformation of $P(\lambda)$ and $\widetilde{P}(\lambda)$.

Proof. Because $P(\lambda)$ and $\widetilde{P}(\lambda)$ are isospectral, they have the same finite and infinite Jordan forms, and so $Q^{-1}(A \lambda-B) R=\widetilde{Q}^{-1}(\widetilde{A} \lambda-\widetilde{B}) \widetilde{R}$ from $(2.10)$, where $Q$ and $R$ are computed according to the procedure outlined in Theorem 2.1 and Lemma 2.2. Note that $\widetilde{Q}$ and $\widetilde{R}$ are obtained by the same process, with every occurrence of $A$ and $B$ in the procedure replaced by $\widetilde{A}$ and $\widetilde{B}$, respectively. With some rearranging, we obtain $\left(\widetilde{Q} Q^{-1}\right)(A \lambda-B)=(\widetilde{A} \lambda-\widetilde{B})\left(\widetilde{R} R^{-1}\right)$, and thus, by (1.1), $\widetilde{Q} Q^{-1}=U$ and $\widetilde{R} R^{-1}=V$ define a structure-preserving transformation of $P(\lambda)$ and $\widetilde{P}(\lambda)$.

2.2.1. The first companion form. As far as we are aware, for any given companion form of a polynomial $P(\lambda)$, the transformation matrices $Q$ and $R$ in (2.10) generally do not have convenient structures independent of the process described in Theorem 2.1 and Lemma 2.2. One known exception is for the first companion form; from Chapter 7 of [8], it turns out that $Q$ and $R$ can be constructed from the coefficients and spectral data of $P(\lambda)$ and its reversal.

Suppose $P(\lambda)$ has finite Jordan form $J_{F} \in \mathbb{C}^{n_{F} \times n_{F}}$ and infinite Jordan form $J_{\infty} \in \mathbb{C}^{n_{\infty} \times n_{\infty}}$, where $n_{F}+n_{\infty}=\ell n$. Let the matrix $V_{F} \in \mathbb{C}^{n \times n_{F}}$ contain the Jordan chains associated with the finite eigenvalues of $P(\lambda)$; the arrangement of the Jordan chains in $V_{F}$ is conformable to the structure of $J_{F}$. A Jordan chain $v_{i j}^{k}\left(k=1,2, \ldots, p_{i j}\right)$ for a distinct finite eigenvalue $\lambda_{i}$ with $j$ th partial multiplicity $p_{i j}$ satisfies the system 
of equations $[8$, p. 25]

$$
\left[\begin{array}{cccc}
P\left(\lambda_{i}\right) & 0 & \cdots & 0 \\
P^{\prime}\left(\lambda_{i}\right) & P\left(\lambda_{i}\right) & \cdots & 0 \\
\vdots & \vdots & \ddots & \vdots \\
\frac{P^{\left(p_{i j}-1\right)}\left(\lambda_{i}\right)}{\left(p_{i j}-1\right) !} & \frac{P^{\left(p_{i j}-2\right)}\left(\lambda_{i}\right)}{\left(p_{i j}-2\right) !} & \cdots & P\left(\lambda_{i}\right)
\end{array}\right]\left[\begin{array}{c}
v_{i j}^{1} \\
v_{i j}^{2} \\
\vdots \\
v_{i j}^{p_{i j}}
\end{array}\right]=0
$$

where $v_{i j}^{1} \neq 0$ is an eigenvector and $v_{i j}^{m}\left(m=2,3, \ldots, p_{i j}\right)$ are generalized eigenvectors. Note that when the polynomial's degree $\ell>1$, the vectors of a Jordan chain do not need to be linearly independent, and the zero vector is an acceptable generalized eigenvector [8, p. 24]. Similarly, let the matrix $V_{\infty} \in \mathbb{C}^{n \times n_{\infty}}$ be populated, conformable to $J_{\infty}$, with the Jordan chains for the zero eigenvalue of the reverse polynomial $P_{\text {rev }}(\lambda)$. If $v_{\infty j}^{k}\left(k=1,2, \ldots, p_{\infty j}\right)$ form a Jordan chain for the zero eigenvalue with $j$ th partial multiplicity $p_{\infty j}$, then

$$
\left[\begin{array}{cccc}
P_{\mathrm{rev}}(0) & 0 & \cdots & 0 \\
P_{\mathrm{rev}}^{\prime}(0) & P_{\mathrm{rev}}(0) & \cdots & 0 \\
\vdots & \vdots & \ddots & \vdots \\
\frac{P_{\mathrm{rev}}^{\left(p_{\infty j}-1\right)}(0)}{\left(p_{\infty j}-1\right) !} & \frac{P_{\mathrm{rev}}^{\left(p_{\infty j}-2\right)}(0)}{\left(p_{\infty j}-2\right) !} & \cdots & P_{\mathrm{rev}}(0)
\end{array}\right]\left[\begin{array}{c}
v_{\infty j}^{1} \\
v_{\infty j}^{2} \\
\vdots \\
v_{\infty j j}^{p_{\infty j}}
\end{array}\right]=0
$$

where $v_{\infty j}^{1} \neq 0$ is an eigenvector and $v_{\infty j}^{m}\left(m=2,3, \ldots, p_{\infty j}\right)$ are generalized eigenvectors. The matrix pairs $\left(V_{F}, J_{F}\right)$ and $\left(V_{\infty}, J_{\infty}\right)$ are known as, respectively, a finite Jordan pair of $P(\lambda)$ and an infinite Jordan pair of the same polynomial [8, pp. 184-185].

Now, by Theorem 7.3 of [8], the finite and infinite Jordan pairs comprise what is termed a decomposable pair of $P(\lambda),\left(\left[\begin{array}{ll}V_{F} & V_{\infty}\end{array}\right], J_{F} \oplus J_{\infty}\right)$. A decomposable pair is a generalization of the familiar standard pair of a matrix polynomial with nonsingular leading coefficient; we refer the reader to Section 7.3 of [8] for a complete description. Because we can generate a decomposable pair of $P(\lambda)$ from its corresponding finite and infinite spectral information, Theorem 7.6 of [8] provides the analogue of (2.10) applied to the first companion form. For our representation of the first companion form in (2.6), it follows from Theorem 7.6 that

$$
Q=\left[\begin{array}{cc}
A_{\ell} V_{F} J_{F}^{\ell-1} & -\sum_{i=0}^{\ell-1} A_{i} V_{\infty} J_{\infty}^{\ell-1-i} \\
V_{F} J_{F}^{\ell-2} & V_{\infty} \\
V_{F} J_{F}^{\ell-3} & V_{\infty} J_{\infty} \\
\vdots & \vdots \\
V_{F} & V_{\infty} J_{\infty}^{\ell-2}
\end{array}\right] \quad \text { and } \quad R=\left[\begin{array}{cc}
V_{F} J_{F}^{\ell-1} & V_{\infty} \\
V_{F} J_{F}^{\ell-2} & V_{\infty} J_{\infty} \\
\vdots & \vdots \\
V_{F} & V_{\infty} J_{\infty}^{\ell-1}
\end{array}\right]
$$

where the invertibility of $Q$ and $R$ are addressed in the proofs of Theorem 7.6 and Theorem 7.3, respectively.

2.3. Parameterizing the structure-preserving transformation. Having shown how to construct a structure-preserving transformation $(U, V)$ of two isospectral matrix polynomials $P(\lambda)$ and $\widetilde{P}(\lambda)$, we now wish to parameterize this transformation through the commutant $S$ of their companion forms' common Weierstrass canonical form. That is, for two given isospectral polynomials, we seek an invertible mapping that relates a nonsingular matrix $S \in \Phi$ to nonsingular matrices $U$ and $V$ that satisfy $U(A \lambda-B)=(\widetilde{A} \lambda-\widetilde{B}) V$. We shall first demonstrate that the commutant $S$ has a special block-diagonal structure. 
Electronic Journal of Linear Algebra, ISSN 1081-3810

A publication of the International Linear Algebra Society

Volume 36, pp. 723-743, November 2020 .

Proposition 2.4. Let $P(\lambda)$ be an $n \times n$ matrix polynomial of degree $\ell$ that is $\Gamma$-regular, has nonzero leading coefficient, and has finite Jordan form $J_{F} \in \mathbb{C}^{n_{F} \times n_{F}}$ and infinite Jordan form $J_{\infty} \in \mathbb{C}^{n_{\infty} \times n_{\infty}}$, where $n_{F}+n_{\infty}=\ell n$. Define the Cauchy contour $\Gamma$ such that its inner domain contains the finite eigenvalues of $P(\lambda)$. Denote the Weierstrass canonical form of any strong linearization of $P(\lambda)$ as $\mathfrak{W}(\lambda)=\left(I \lambda-J_{F}\right) \oplus$ $\left(J_{\infty} \lambda-I\right)$, and let $S \in \mathbb{C}^{\ell n \times \ell n}$. Then $S \mathfrak{W}(\lambda)=\mathfrak{W}(\lambda) S$ if and only if

$$
S=S_{F} \oplus S_{\infty}, \quad \text { where } S_{F} J_{F}=J_{F} S_{F} \quad \text { and } \quad S_{\infty} J_{\infty}=J_{\infty} S_{\infty}
$$

Proof. First, suppose $S$ is as given by (2.15). Consequently,

$$
S \mathfrak{W}(\lambda)=S_{F}\left(I \lambda-J_{F}\right) \oplus S_{\infty}\left(J_{\infty} \lambda-I\right)=\left(I \lambda-J_{F}\right) S_{F} \oplus\left(J_{\infty} \lambda-I\right) S_{\infty}=\mathfrak{W}(\lambda) S
$$

Next, suppose $S \mathfrak{W}(\lambda)=\mathfrak{W}(\lambda) S$, where $S=\left[\begin{array}{ll}S_{1} & S_{2} \\ S_{3} & S_{4}\end{array}\right]$ with $S_{1} \in \mathbb{C}^{n_{F} \times n_{F}}$ and $S_{4} \in \mathbb{C}^{n_{\infty} \times n_{\infty}}$. As a result, we obtain the expressions

$$
\begin{array}{lll}
S_{1} \lambda-S_{1} J_{F}=S_{1} \lambda-J_{F} S_{1} & \Rightarrow & S_{1} J_{F}=J_{F} S_{1}, \\
S_{2} J_{\infty} \lambda-S_{2}=S_{2} \lambda-J_{F} S_{2} & \Rightarrow & S_{2} J_{\infty}=S_{2} \quad \text { and } \quad S_{2}=J_{F} S_{2}, \\
S_{3} \lambda-S_{3} J_{F}=J_{\infty} S_{3} \lambda-S_{3} & \Rightarrow & S_{3}=J_{\infty} S_{3} \quad \text { and } \quad S_{3} J_{F}=S_{3}, \\
S_{4} J_{\infty} \lambda-S_{4}=J_{\infty} S_{4} \lambda-S_{4} & \Rightarrow & S_{4} J_{\infty}=J_{\infty} S_{4} .
\end{array}
$$

Now, from (2.17), the matrix $S_{2} \in \mathbb{C}^{n_{F} \times n_{\infty}}$ must satisfy $S_{2} J_{\infty}=I_{n_{F}} S_{2}$, where we have purposefully included the $n_{F} \times n_{F}$ identity matrix $I_{n_{F}}$. Because the infinite Jordan form $J_{\infty}$ comprises only Jordan blocks of a zero eigenvalue, the spectra of $J_{\infty}$ and $I_{n_{F}}$ are disjoint. Therefore, from Section 1 of Chapter VIII in [5], we must have $S_{2}=0$, which also satisfies the second condition $S_{2}=J_{F} S_{2}$ in (2.17). By the same reasoning, the only solution $S_{3} \in \mathbb{C}^{n_{\infty} \times n_{F}}$ to $S_{3} I_{n_{F}}=J_{\infty} S_{3}$ in (2.18) is $S_{3}=0$, and thus, the second condition $S_{3} J_{F}=S_{3}$ is also satisfied. Consequently, $S$ must be a block-diagonal matrix: $S=S_{1} \oplus S_{4}$, where the blocks $S_{1}$ and $S_{4}$ are such that (2.16) and (2.19) hold, which coincides with (2.15) after some relabeling.

Next, what is the dimension of the linear space $\Phi$ defined in (1.2)? Let $\lambda_{i}(i=1,2, \ldots, s)$ be the $s$ distinct finite eigenvalues of a polynomial $P(\lambda)$ with partial multiplicities $p_{i j}\left(j=1,2, \ldots, g_{i}\right)$, where $p_{i 1} \geq p_{i 2} \geq \cdots \geq p_{i g_{i}}$. From (2.15) of Proposition 2.4, the matrix $S_{F}$ in the block-diagonal commutant $S=S_{F} \oplus S_{\infty}$ commutes with the finite Jordan form $J_{F}$ common to $P(\lambda)$ and a polynomial $\widetilde{P}(\lambda)$ isospectral to it. Thus, we know that $S_{F}$ is a matrix of $s$ blocks on its diagonal, consistent with the arrangement of the Jordan blocks for $\lambda_{i}$ on the diagonal of $J_{F}$, where each of the $s$ blocks in $S_{F}$ contains submatrices with triangular Toeplitz structure (e.g., see Section 1 and Section 2 of Chapter VIII in [5] and Section 9.1 of [7]). From $[5,11]$, the number of independent arbitrary parameters present in $S_{F}$ is given by

$$
N_{F}=\sum_{i=1}^{s} \sum_{j=1}^{g_{i}}(2 j-1) p_{i j}
$$

Now, suppose the infinite eigenvalue of $P(\lambda)$ has partial multiplicities $p_{\infty j}\left(j=1,2, \ldots, g_{\infty}\right)$, with $p_{\infty 1} \geq$ $p_{\infty 2} \geq \cdots \geq p_{\infty g_{\infty}}$. By $(2.15)$, the matrix $S_{\infty}$ commutes with the polynomials' shared infinite Jordan form $J_{\infty}$, and so $S_{\infty}$ is similarly composed of submatrices with triangular Toeplitz structure. Therefore, analogous to $(2.20), S_{\infty}$ contains

$$
N_{\infty}=\sum_{j=1}^{g_{\infty}}(2 j-1) p_{\infty j}
$$


free parameters. Finally, because the matrix $S \in \Phi$ has the block-diagonal structure $S=S_{F} \oplus S_{\infty}$, it comprises $N_{F}+N_{\infty}$ independent parameters, making the dimension of the linear space $\Phi$ also $N_{F}+N_{\infty}$.

Moving on, denote the set of all structure-preserving transformations $(U, V)$ relating two isospectral polynomials $P(\lambda)$ and $\widetilde{P}(\lambda)$ as $\Xi$ :

$$
\Xi:=\left\{(U, V) \in \mathbb{C}^{\ell n \times \ell n} \times \mathbb{C}^{\ell n \times \ell n}: U(A \lambda-B)=(\widetilde{A} \lambda-\widetilde{B}) V\right\},
$$

which is a linear space. We then arrive at the following statement that is a direct generalization of Theorem 2.2 in [11].

Theorem 2.5. Let $P(\lambda)$ and $\widetilde{P}(\lambda)$ be $n \times n$ matrix polynomials of degree $\ell$ that are isospectral and $\Gamma$-regular, have nonzero leading coefficients, and have shared finite Jordan form $J_{F} \in \mathbb{C}^{n_{F} \times n_{F}}$ and infinite Jordan form $J_{\infty} \in \mathbb{C}^{n_{\infty} \times n_{\infty}}$, where $n_{F}+n_{\infty}=\ell n$. Define the Cauchy contour $\Gamma$ such that its inner domain contains the finite eigenvalues of $P(\lambda)$. Let $A \lambda-B$ and $\widetilde{A} \lambda-\widetilde{B}$ be companion forms of $P(\lambda)$ and $\widetilde{P}(\lambda)$, respectively. Define the sets $\Phi$ and $\Xi$ according to (1.2) and (2.22), respectively. Let $Q$ and $R$ be the invertible matrices of (2.11) that satisfy (2.10), and similarly for $\widetilde{Q}$ and $\widetilde{R}$. Then the mapping $\psi: \Phi \rightarrow \Xi$ defined by

$$
\psi(S)=\left(\widetilde{Q} S Q^{-1}, \widetilde{R} S R^{-1}\right)
$$

is an isomorphism of linear spaces with dimension $N_{F}+N_{\infty}$, where $N_{F}$ and $N_{\infty}$ are given by (2.20) and (2.21), respectively.

Proof. First, note from (2.10) that

$$
Q^{-1} A R=\left[\begin{array}{cc}
I & 0 \\
0 & J_{\infty}
\end{array}\right] \text { and } Q^{-1} B R=\left[\begin{array}{cc}
J_{F} & 0 \\
0 & I
\end{array}\right] .
$$

Now, if $U=\widetilde{Q} S Q^{-1}$ and $V=\widetilde{R} S R^{-1}$, where $S \in \Phi$ must have the block-diagonal structure in (2.15) by Proposition 2.4, then repeated use of (2.23) reveals that

$$
\begin{array}{rlrl}
U A & =\left(\widetilde{Q} S Q^{-1}\right) A & U B & =\left(\widetilde{Q} S Q^{-1}\right) B \\
& =\widetilde{Q} S\left(Q^{-1} A R\right) R^{-1} & & \\
& =\widetilde{Q}\left[\begin{array}{cc}
S_{F} & 0 \\
0 & S_{\infty}
\end{array}\right]\left[\begin{array}{cc}
I & 0 \\
0 & J_{\infty}
\end{array}\right] R^{-1} & & =\widetilde{Q}\left[\begin{array}{cc}
S_{F} & 0 \\
0 & S_{\infty}
\end{array}\right]\left[\begin{array}{cc}
J_{F} & 0 \\
0 & I
\end{array}\right] R^{-1} \\
& =\widetilde{Q}\left[\begin{array}{cc}
I & 0 \\
0 & J_{\infty}
\end{array}\right]\left[\begin{array}{cc}
S_{F} & 0 \\
0 & S_{\infty}
\end{array}\right] R^{-1} & & =\widetilde{Q}\left[\begin{array}{cc}
J_{F} & 0 \\
0 & I
\end{array}\right]\left[\begin{array}{cc}
S_{F} & 0 \\
0 & S_{\infty}
\end{array}\right] R^{-1} \\
& =\widetilde{Q}(\widetilde{Q}-1 \widetilde{A} \widetilde{R}) S R^{-1} & & =\widetilde{Q}(\widetilde{Q}-1 \widetilde{B} \widetilde{R}) S R^{-1} \\
& =\widetilde{A}\left(\widetilde{R} S R^{-1}\right) & & =\widetilde{B}\left(\widetilde{R} S R^{-1}\right) \\
& =\widetilde{A} V, & & =\widetilde{B} V .
\end{array}
$$

Consequently, $\left(\widetilde{Q} S Q^{-1}\right)(A \lambda-B)=(\widetilde{A} \lambda-\widetilde{B})\left(\widetilde{R} S R^{-1}\right)$, and so $\left(\widetilde{Q} S Q^{-1}, \widetilde{R} S R^{-1}\right) \in \Xi$. Thus, the mapping $\psi$ is well defined.

Next, we show that the mapping $\psi$ is bijective. Because the transformation matrices $Q, R, \widetilde{Q}$, and $\widetilde{R}$ are invertible, $\psi$ is injective. To demonstrative surjectivity of $\psi$, start by letting some $(U, V) \in \Xi$ so that $U(A \lambda-B)=(\widetilde{A} \lambda-\widetilde{B}) V$. Apply $(2.23)_{1}$ to $U A=\widetilde{A} V$ and rearrange the result to obtain

$$
\left(\widetilde{Q}^{-1} U Q\right)\left[\begin{array}{cc}
I & 0 \\
0 & J_{\infty}
\end{array}\right]=\left[\begin{array}{cc}
I & 0 \\
0 & J_{\infty}
\end{array}\right]\left(\widetilde{R}^{-1} V R\right)
$$


Electronic Journal of Linear Algebra, ISSN 1081-3810

A publication of the International Linear Algebra Society

Volume 36, pp. 723-743, November 2020.

Likewise, manipulating $U B=\widetilde{B} V$ after applying $(2.23)_{2}$ yields

$$
\left(\widetilde{Q}^{-1} U Q\right)\left[\begin{array}{cc}
J_{F} & 0 \\
0 & I
\end{array}\right]=\left[\begin{array}{cc}
J_{F} & 0 \\
0 & I
\end{array}\right]\left(\widetilde{R}^{-1} V R\right)
$$

Now, let $\widetilde{Q}^{-1} U Q=\left[\begin{array}{ll}W_{1} & W_{2} \\ W_{3} & W_{4}\end{array}\right]$ and $\widetilde{R}^{-1} V R=\left[\begin{array}{cc}X_{1} & X_{2} \\ X_{3} & X_{4}\end{array}\right]$, where $W_{1}, X_{1} \in \mathbb{C}^{n_{F} \times n_{F}}$ and $W_{4}, X_{4} \in \mathbb{C}_{\infty} \times n_{\infty}$. Therefore, from (2.24) and (2.25), respectively,

$$
\left[\begin{array}{ll}
W_{1} & W_{2} J_{\infty} \\
W_{3} & W_{4} J_{\infty}
\end{array}\right]=\left[\begin{array}{cc}
X_{1} & X_{2} \\
J_{\infty} X_{3} & J_{\infty} X_{4}
\end{array}\right] \text { and } \quad\left[\begin{array}{ll}
W_{1} J_{F} & W_{2} \\
W_{3} J_{F} & W_{4}
\end{array}\right]=\left[\begin{array}{cc}
J_{F} X_{1} & J_{F} X_{2} \\
X_{3} & X_{4}
\end{array}\right]
$$

We immediately see in (2.26) that $W_{1}=X_{1}$ and $W_{4}=X_{4}$ (i.e., $\widetilde{Q}^{-1} U Q$ and $\widetilde{R}^{-1} V R$ have the same blocks on their diagonals), and hence,

$$
W_{1} J_{F}=J_{F} W_{1} \quad \text { and } \quad W_{4} J_{\infty}=J_{\infty} W_{4}
$$

Also note from (2.26) that

$$
X_{2}=W_{2} J_{\infty} \quad \text { and } \quad W_{2}=J_{F} X_{2}
$$

Let $w_{2 i}\left(i=1,2, \ldots, n_{\infty}\right)$ denote the $n_{F}$-dimensional columns of $W_{2} \in \mathbb{C}^{n_{F} \times n_{\infty}}$. At worst, $J_{\infty}$ is a single $n_{\infty} \times n_{\infty}$ Jordan block with eigenvalue 0 , and so, from $(2.28)_{1}$,

$$
X_{2}=\left[\begin{array}{lllll}
w_{21} & w_{22} & w_{23} & \cdots & w_{2 n_{\infty}}
\end{array}\right]\left[\begin{array}{ccccc}
0 & 1 & 0 & \cdots & 0 \\
0 & 0 & 1 & \cdots & 0 \\
\vdots & \vdots & \vdots & \ddots & \vdots \\
0 & 0 & 0 & \cdots & 1 \\
0 & 0 & 0 & \cdots & 0
\end{array}\right]=\left[\begin{array}{llllll}
0 & w_{21} & w_{22} & \cdots & w_{2\left(n_{\infty}-1\right)}
\end{array}\right] \in \mathbb{C}^{n_{F} \times n_{\infty}} .
$$

We then find from $(2.28)_{2}$ that

$$
\begin{aligned}
{\left[\begin{array}{lllll}
w_{21} & w_{22} & w_{23} & \cdots & w_{2 n_{\infty}}
\end{array}\right] } & =J_{F}\left[\begin{array}{lllll}
0 & w_{21} & w_{22} & \cdots & w_{2\left(n_{\infty}-1\right)}
\end{array}\right] \\
& =\left[\begin{array}{llllll}
0 & J_{F} w_{21} & J_{F} w_{22} & \cdots & J_{F} w_{2\left(n_{\infty}-1\right)}
\end{array}\right] .
\end{aligned}
$$

The result $w_{21}=0$ begins a chain reaction leading to $w_{22}=w_{23}=\cdots=w_{2 n_{\infty}}=0$, and thus, $W_{2}=X_{2}=0$. Returning to $(2.26)$, we also have the following conditions relating $W_{3} \in \mathbb{C}^{n_{\infty} \times n_{F}}$ and $X_{3} \in \mathbb{C}^{n_{\infty} \times n_{F}}$ :

$$
W_{3}=J_{\infty} X_{3} \quad \text { and } \quad X_{3}=W_{3} J_{F}
$$

Let $x_{3 i}$ be the $n_{F}$-dimensional rows of $X_{3}$ so that, from $(2.29)_{1}$,

$$
W_{3}=\left[\begin{array}{ccccc}
0 & 1 & 0 & \cdots & 0 \\
0 & 0 & 1 & \cdots & 0 \\
\vdots & \vdots & \vdots & \ddots & \vdots \\
0 & 0 & 0 & \cdots & 1 \\
0 & 0 & 0 & \cdots & 0
\end{array}\right]\left[\begin{array}{c}
x_{31} \\
x_{32} \\
\vdots \\
x_{3\left(n_{\infty}-1\right)} \\
x_{3 n_{\infty}}
\end{array}\right]=\left[\begin{array}{c}
x_{32} \\
x_{33} \\
\vdots \\
x_{3 n_{\infty}} \\
0
\end{array}\right]
$$


Therefore, $(2.29)_{2}$ yields

$$
\left[\begin{array}{c}
x_{31} \\
x_{32} \\
\vdots \\
x_{3\left(n_{\infty}-1\right)} \\
x_{3 n_{\infty}}
\end{array}\right]=\left[\begin{array}{c}
x_{32} \\
x_{33} \\
\vdots \\
x_{3 n_{\infty}} \\
0
\end{array}\right] J_{F}=\left[\begin{array}{c}
x_{32} J_{F} \\
x_{33} J_{F} \\
\vdots \\
x_{3 n_{\infty}} J_{F} \\
0
\end{array}\right]
$$

Similar to the case of $W_{2}$ and $X_{2}$, we see that $x_{3 n_{\infty}}=0$ initiates a chain reaction resulting in $x_{3\left(n_{\infty}-1\right)}=$ $x_{3\left(n_{\infty}-2\right)}=\cdots=x_{31}=0$, and so $W_{3}=X_{3}=0$ as well. In general, $J_{\infty}$ is an $n_{\infty} \times n_{\infty}$ block-diagonal matrix of zero-eigenvalue Jordan blocks, so any $J_{\infty}$ can be formed by appropriately removing 1's from the superdiagonal of a single $n_{\infty} \times n_{\infty}$ Jordan block. Thus, $W_{2}, W_{3}, X_{2}$, and $X_{3}$ will always be zero matrices, regardless of the number and sizes of the Jordan blocks that comprise $J_{\infty}$. Consequently, $\widetilde{Q}^{-1} U Q$ and $\widetilde{R}^{-1} V R$ are identical block-diagonal matrices: $\widetilde{Q}^{-1} U Q=\widetilde{R}^{-1} V R=S_{F} \oplus S_{\infty}$, with some renaming, where (2.27) requires that $S_{F} J_{F}=J_{F} S_{F}$ and $S_{\infty} J_{\infty}=J_{\infty} S_{\infty}$. Taking $S=S_{F} \oplus S_{\infty}$ and recalling Proposition 2.4, we then have $S \in \Phi, U=\widetilde{Q} S Q^{-1}$, and $V=\widetilde{R} S R^{-1}$, as desired.

Thus, so long as the commutant $S=S_{F} \oplus S_{\infty}$ is chosen to be invertible, the matrices $U$ and $V$ will also be invertible, and hence, $(U, V)$ will be a structure-preserving transformation of two isospectral matrix polynomials. What is the general form of $S$ ? First, for the $s$ distinct finite eigenvalues $\lambda_{i}(i=1,2, \ldots, s)$ with partial multiplicities $p_{i j}\left(j=1,2, \ldots, g_{i}\right)$ arranged by decreasing size, construct the finite Jordan form $J_{F}$ according to

$$
J_{F}=\bigoplus_{i=1}^{s}\left(\bigoplus_{j=1}^{g_{i}} J_{i j}\right), \quad \text { where } J_{i j}=\left[\begin{array}{ccccc}
\lambda_{i} & 1 & 0 & \cdots & 0 \\
0 & \lambda_{i} & 1 & \cdots & 0 \\
\vdots & \vdots & \ddots & \ddots & \vdots \\
0 & 0 & \cdots & \lambda_{i} & 1 \\
0 & 0 & \cdots & 0 & \lambda_{i}
\end{array}\right] \in \mathbb{C}^{p_{i j} \times p_{i j}}
$$

Next, for the eigenvalue at infinity with partial multiplicities $p_{\infty j}\left(j=1,2, \ldots, g_{\infty}\right)$, also ordered from largest to smallest, build the infinite Jordan form $J_{\infty}$ from Jordan blocks with eigenvalue 0 as follows:

$$
J_{\infty}=\bigoplus_{j=1}^{g_{\infty}} J_{\infty j}, \quad \text { where } J_{\infty j}=\left[\begin{array}{ccccc}
0 & 1 & 0 & \cdots & 0 \\
0 & 0 & 1 & \cdots & 0 \\
\vdots & \vdots & \vdots & \ddots & \vdots \\
0 & 0 & 0 & \cdots & 1 \\
0 & 0 & 0 & \cdots & 0
\end{array}\right] \in \mathbb{R}^{p_{\infty j} \times p_{\infty j}}
$$

As mentioned earlier, because $S_{F}$ and $S_{\infty}$ commute with the Jordan forms $J_{F}$ and $J_{\infty}$, respectively, these components of the commutant $S$ are composed of submatrices with triangular Toeplitz structure. Specifically (see Section 1 and Section 2 of Chapter VIII in [5] and Section 9.1 of [7]), for the finite eigenvalues,

$$
S_{F}=\bigoplus_{i=1}^{s} S_{i}, \quad \text { where } \quad S_{i}=\left[S_{i, j k}\right] \quad\left(j, k=1,2, \ldots, g_{i}\right)
$$


Electronic Journal of Linear Algebra, ISSN 1081-3810

A publication of the International Linear Algebra Society

Volume 36, pp. 723-743, November 2020 .

733

Parameterized Structure-preserving Transformations of Matrix Polynomials

and

$$
\begin{aligned}
& S_{i, j k}=\left[\begin{array}{cccc}
1 \alpha_{i, j k} & { }_{2} \alpha_{i, j k} & \cdots & p_{i k} \alpha_{i, j k} \\
0 & { }_{1} \alpha_{i, j k} & \cdots & p_{i k}-1 \alpha_{i, j k} \\
\vdots & \vdots & \ddots & \vdots \\
0 & 0 & \cdots & 1 \alpha_{i, j k} \\
0 & 0 & \cdots & 0 \\
\vdots & \vdots & & \vdots \\
0 & 0 & \cdots & 0
\end{array}\right] \in \mathbb{C}^{p_{i j} \times p_{i k}} \quad \text { if } j<k \\
& S_{i, j k}=\left[\begin{array}{cccc}
1 \alpha_{i, j k} & { }_{2} \alpha_{i, j k} & \cdots & p_{i j} \alpha_{i, j k} \\
0 & { }_{1} \alpha_{i, j k} & \cdots & p_{i j}-1 \alpha_{i, j k} \\
\vdots & \vdots & \ddots & \vdots \\
0 & 0 & \cdots & { }_{1} \alpha_{i, j k}
\end{array}\right] \in \mathbb{C}^{p_{i j} \times p_{i j}} \quad \text { if } j=k \\
& S_{i, j k}=\left[\begin{array}{ccccccc}
0 & \cdots & 0 & { }_{1} \alpha_{i, j k} & { }_{2} \alpha_{i, j k} & \cdots & p_{i j} \alpha_{i, j k} \\
0 & \cdots & 0 & 0 & { }_{1} \alpha_{i, j k} & \cdots & p_{i j}-1 \alpha_{i, j k} \\
\vdots & & \vdots & \vdots & \vdots & \ddots & \vdots \\
0 & \cdots & 0 & 0 & 0 & \cdots & { }_{1} \alpha_{i, j k}
\end{array}\right] \in \mathbb{C}^{p_{i j} \times p_{i k}} \quad \text { if } j>k .
\end{aligned}
$$

We are free to choose the values of the parameters ${ }_{m} \alpha_{i, j k} \in \mathbb{C}$. Likewise, $S_{\infty}=\left[S_{\infty, j k}\right]\left(j, k=1,2, \ldots, g_{\infty}\right)$ for the eigenvalue at infinity, where $S_{\infty, j k}$ are constructed in the same manner as $S_{i, j k}$ in $(2.33)-(2.35)$.

We next illustrate with a simple example the process for generating a parameterized structure-preserving transformation that connects two isospectral polynomials.

2.4. An example. Consider the cubic matrix polynomial

$$
P(\lambda)=A_{3} \lambda^{3}+A_{2} \lambda^{2}+A_{1} \lambda+A_{0}=\left[\begin{array}{ll}
1 & 0 \\
1 & 0
\end{array}\right] \lambda^{3}+\left[\begin{array}{cc}
1 & 1 \\
-1 & 1
\end{array}\right] \lambda^{2}+\left[\begin{array}{ll}
1 & 1 \\
0 & 0
\end{array}\right] \lambda+\left[\begin{array}{ll}
1 & 0 \\
0 & 0
\end{array}\right],
$$

which has two distinct finite eigenvalues that each form a $2 \times 2$ Jordan block: $-1\left(g_{1}=1\right.$ and $\left.p_{11}=2\right)$ and $0\left(g_{2}=1\right.$ and $\left.p_{21}=2\right)$. The eigenvalue at infinity happens to form a $2 \times 2$ Jordan block as well $\left(g_{\infty}=1\right.$ and $\left.p_{\infty 1}=2\right)$. Thus, using $(2.30)$ and $(2.31)$,

$$
J_{F}=\left[\begin{array}{cc}
-1 & 1 \\
0 & -1
\end{array}\right] \oplus\left[\begin{array}{ll}
0 & 1 \\
0 & 0
\end{array}\right]=\left[\begin{array}{cccc}
-1 & 1 & 0 & 0 \\
0 & -1 & 0 & 0 \\
0 & 0 & 0 & 1 \\
0 & 0 & 0 & 0
\end{array}\right] \quad \text { and } \quad J_{\infty}=\left[\begin{array}{ll}
0 & 1 \\
0 & 0
\end{array}\right]
$$

are, respectively, finite and infinite Jordan forms of $P(\lambda)$. The polynomial

$$
\widetilde{P}(\lambda)=\widetilde{A}_{3} \lambda^{3}+\widetilde{A}_{2} \lambda^{2}+\widetilde{A}_{1} \lambda+\widetilde{A}_{0}=\left[\begin{array}{ll}
1 & 0 \\
0 & 0
\end{array}\right] \lambda^{3}+\left[\begin{array}{cc}
1 & 1 \\
-1 & 0
\end{array}\right] \lambda^{2}+\left[\begin{array}{cc}
0 & 0 \\
-1 & 0
\end{array}\right] \lambda+\left[\begin{array}{ll}
0 & 0 \\
0 & 1
\end{array}\right]
$$

is isospectral to $P(\lambda)$. We now follow the process described in Theorem 2.1 and Lemma 2.2 to generate the matrices that comprise a parameterized structure-preserving transformation of $P(\lambda)$ and $\widetilde{P}(\lambda)$. In this 
example, our strong linearization of choice is the Fiedler pencil

$$
C_{F_{\sigma}}(\lambda)=M_{3} \lambda-M_{2} M_{0} M_{1}=\left[\begin{array}{ccc}
A_{3} & 0 & 0 \\
0 & I & 0 \\
0 & 0 & I
\end{array}\right] \lambda-\left[\begin{array}{ccc}
-A_{2} & -A_{1} & I \\
I & 0 & 0 \\
0 & -A_{0} & 0
\end{array}\right],
$$

in which case

$$
A \lambda-B=\left[\begin{array}{cccccc}
1 & 0 & 0 & 0 & 0 & 0 \\
1 & 0 & 0 & 0 & 0 & 0 \\
0 & 0 & 1 & 0 & 0 & 0 \\
0 & 0 & 0 & 1 & 0 & 0 \\
0 & 0 & 0 & 0 & 1 & 0 \\
0 & 0 & 0 & 0 & 0 & 1
\end{array}\right] \lambda-\left[\begin{array}{cccccc}
-1 & -1 & -1 & -1 & 1 & 0 \\
1 & -1 & 0 & 0 & 0 & 1 \\
1 & 0 & 0 & 0 & 0 & 0 \\
0 & 1 & 0 & 0 & 0 & 0 \\
0 & 0 & -1 & 0 & 0 & 0 \\
0 & 0 & 0 & 0 & 0 & 0
\end{array}\right]
$$

is the corresponding companion form of $P(\lambda)$. Using any Cauchy contour $\Gamma$ whose inner domain contains the finite eigenvalues -1 and 0 , we find from (2.8) and (2.9) that

$$
X=\left[\begin{array}{cccccc}
-3 & 2 & -1 & -1 & 1 & -1 \\
-8 & 3 & -3 & -4 & 4 & -2 \\
1 & -1 & 1 & 0 & 0 & 0 \\
4 & -2 & 1 & 2 & -1 & 1 \\
0 & 0 & 0 & 0 & 1 & 0 \\
0 & 0 & 0 & 0 & 0 & 1
\end{array}\right] \quad \text { and } \quad W=\left[\begin{array}{cccccc}
-2 & 1 & -1 & -1 & 1 & -1 \\
-2 & 1 & -1 & -1 & 1 & -1 \\
1 & -1 & 1 & 0 & 0 & 0 \\
3 & -1 & 1 & 2 & -1 & 1 \\
0 & 0 & 0 & 0 & 1 & 0 \\
0 & 0 & 0 & 0 & 0 & 1
\end{array}\right],
$$

where $\operatorname{rank}(W)=4$. We then use Gaussian elimination to determine bases for the image and kernel of $W$ :

$$
Y=\left[\begin{array}{cccc|cc}
-2 & 1 & 1 & -1 & 0 & -1 \\
-2 & 1 & 1 & -1 & 1 & -1 \\
1 & -1 & 0 & 0 & 1 & 0 \\
3 & -1 & -1 & 1 & 0 & 1 \\
0 & 0 & 1 & 0 & 0 & 0 \\
0 & 0 & 0 & 1 & 0 & 0
\end{array}\right]
$$

Consequently,

$$
\begin{aligned}
& Y^{-1} A X Y=\left[\begin{array}{cccc|cc}
1 & 0 & 0 & 0 & 0 & 0 \\
0 & 1 & 0 & 0 & 0 & 0 \\
0 & 0 & 1 & 0 & 0 & 0 \\
0 & 0 & 0 & 1 & 0 & 0 \\
\hline 0 & 0 & 0 & 0 & 0 & 0 \\
0 & 0 & 0 & 0 & -1 & 0
\end{array}\right] \\
& Y^{-1} B X Y=\left[\begin{array}{cccc|cc}
-2 & 1 & 1 & 0 & 0 & 0 \\
0 & 0 & 0 & 1 & 0 & 0 \\
-1 & 1 & 0 & 0 & 0 & 0 \\
0 & 0 & 0 & 0 & 0 & 0 \\
\hline 0 & 0 & 0 & 0 & 1 & 0 \\
0 & 0 & 0 & 0 & 0 & 1
\end{array}\right] \\
& =\left[\begin{array}{cc}
I & 0 \\
0 & T_{2}
\end{array}\right] \text {, } \\
& =\left[\begin{array}{cc}
T_{1} & 0 \\
0 & I
\end{array}\right] \text {, }
\end{aligned}
$$

and we find that

$$
Z_{1}=\left[\begin{array}{cccc}
1 & 1 & 1 & -2 \\
0 & 0 & 1 & -1 \\
1 & 2 & 1 & -2 \\
0 & 0 & 0 & 1
\end{array}\right] \text { and } Z_{2}=\left[\begin{array}{cc}
0 & 1 \\
-1 & 0
\end{array}\right]
$$


Electronic Journal of Linear Algebra, ISSN 1081-3810

A publication of the International Linear Algebra Society

Volume 36, pp. 723-743, November 2020 .

result in $Z_{1}^{-1} T_{1} Z_{1}=J_{F}$ and $Z_{2}^{-1} T_{2} Z_{2}=J_{\infty}$. With $Z=Z_{1} \oplus Z_{2}$, evaluating (2.11) gives

$$
Q=\left[\begin{array}{cccccc}
-1 & 0 & 0 & 0 & 1 & 0 \\
-1 & 0 & 0 & 0 & 1 & 1 \\
1 & 1 & 0 & -1 & 0 & 1 \\
2 & 1 & 1 & -2 & -1 & 0 \\
1 & 2 & 1 & -2 & 0 & 0 \\
0 & 0 & 0 & 1 & 0 & 0
\end{array}\right] \quad \text { and } \quad R=\left[\begin{array}{cccccc}
-1 & 0 & 0 & 0 & 0 & 1 \\
-2 & 1 & 0 & 1 & -1 & 0 \\
1 & 1 & 0 & -1 & 0 & 0 \\
2 & 1 & 1 & -2 & 0 & -1 \\
1 & 2 & 1 & -2 & 0 & 0 \\
0 & 0 & 0 & 1 & 0 & 0
\end{array}\right]
$$

We then repeat this process with the corresponding companion form of $\widetilde{P}(\lambda)$,

$$
\widetilde{A} \lambda-\widetilde{B}=\left[\begin{array}{cccccc}
1 & 0 & 0 & 0 & 0 & 0 \\
0 & 0 & 0 & 0 & 0 & 0 \\
0 & 0 & 1 & 0 & 0 & 0 \\
0 & 0 & 0 & 1 & 0 & 0 \\
0 & 0 & 0 & 0 & 1 & 0 \\
0 & 0 & 0 & 0 & 0 & 1
\end{array}\right] \lambda-\left[\begin{array}{cccccc}
-1 & -1 & 0 & 0 & 1 & 0 \\
1 & 0 & 1 & 0 & 0 & 1 \\
1 & 0 & 0 & 0 & 0 & 0 \\
0 & 1 & 0 & 0 & 0 & 0 \\
0 & 0 & 0 & 0 & 0 & 0 \\
0 & 0 & 0 & -1 & 0 & 0
\end{array}\right]
$$

from which we ultimately obtain

$$
\widetilde{Q}=\left[\begin{array}{cccccc}
-1 & -1 & 0 & 1 & 1 & -1 \\
0 & 0 & 0 & 0 & 0 & 1 \\
1 & 2 & 1 & -2 & 0 & 1 \\
0 & -1 & 0 & 1 & -1 & 0 \\
0 & 0 & 0 & 1 & 0 & 0 \\
0 & -1 & -1 & 1 & 0 & 1
\end{array}\right] \quad \text { and } \quad \widetilde{R}=\left[\begin{array}{cccccc}
-1 & -1 & 0 & 1 & 0 & 1 \\
0 & 1 & 0 & 0 & -1 & 0 \\
1 & 2 & 1 & -2 & 0 & 0 \\
0 & -1 & 0 & 1 & 0 & -1 \\
0 & 0 & 0 & 1 & 0 & 0 \\
0 & -1 & -1 & 1 & 0 & 0
\end{array}\right]
$$

Now, according to (2.20) and (2.21), the dimension of $\Phi$ is 6 . Indeed, following (2.32)-(2.35), we have

$$
S_{F}=\left[\begin{array}{cc}
a & b \\
0 & a
\end{array}\right] \oplus\left[\begin{array}{ll}
c & d \\
0 & c
\end{array}\right]=\left[\begin{array}{llll}
a & b & 0 & 0 \\
0 & a & 0 & 0 \\
0 & 0 & c & d \\
0 & 0 & 0 & c
\end{array}\right] \quad \text { and } \quad S_{\infty}=\left[\begin{array}{cc}
e & f \\
0 & e
\end{array}\right]
$$

for the commutant $S=S_{F} \oplus S_{\infty}$, where the 6 free parameters can be any combination of real and nonreal numbers that makes $S$ invertible. Therefore, by Theorem 2.5, all structure-preserving transformations $(U, V)$ connecting the isospectral polynomials $P(\lambda)$ and $\widetilde{P}(\lambda)$ through their respective companion forms (2.36) and (2.37) are computed from

$$
U=\widetilde{Q} S Q^{-1}=\left[\begin{array}{cccccc}
-a+b+4 e-f & a-2 e+f & -a+e & b+e & -b-e & -a+c+e \\
-e & e & 0 & 0 & 0 & 0 \\
-b-e & -a+c+e & a-c & -a-b+c & a+b & a-c+d \\
a-3 e+f & e-f & -e & a-e & -a+e & c-e \\
0 & 0 & 0 & 0 & 0 & c \\
a-e & -c+e & c & a-c & -a & -d
\end{array}\right]
$$


and

$$
V=\widetilde{R} S R^{-1}=\left[\begin{array}{cccccc}
b+2 e & 0 & -a+e & b+e & -b-e & -a+c+e \\
-a+3 e-2 f & e & 2 e-f & -a+3 e-f & a-3 e+f & e-f \\
-a-b+c & 0 & a-c & -a-b+c & a+b & a-c+d \\
a-2 e & 0 & -e & a-e & -a+e & c-e \\
0 & 0 & 0 & 0 & 0 & c \\
a-c & 0 & c & a-c & -a & -d
\end{array}\right]
$$

3. Real matrix polynomials. When two isospectral polynomials $P(\lambda)$ and $\widetilde{P}(\lambda)$ are real, we can use a real commutant $S$ of a real Weierstrass canonical form $\mathfrak{W}(\lambda)=\left(I \oplus J_{\infty}\right) \lambda-\left(J_{F} \oplus I\right)$ to parameterize all real structure-preserving transformations relating $P(\lambda)$ and $\widetilde{P}(\lambda)$. Note that Theorem 2.5 still applies when we restrict our attention to real matrices so long as we use a real finite Jordan form $J_{F}$ of $P(\lambda)$ and $\widetilde{P}(\lambda)$; no change is required for the infinite Jordan form $J_{\infty}$ because it is always real. While no separate statement is needed to accommodate the case of real polynomials, several modifications to our procedure outlined in Section 2.3 must be made.

First, because the nonreal finite eigenvalues for a real polynomial $P(\lambda)$ must occur in conjugate pairs, it is convenient to index the finite eigenvalues as follows. Let $\lambda_{i}(i=1,2, \ldots, r)$ denote the $r$ distinct real eigenvalues, and let $\lambda_{r+j}=\sigma_{r+j}+\mathrm{i} \omega_{r+j}(j=1,2, \ldots, c)$, with $\omega_{r+j}>0$, and $\bar{\lambda}_{r+j}$ be the $2 c$ distinct nonreal eigenvalues that form $c$ conjugate pairs. Associated with each finite eigenvalue $\lambda_{i}(i=1,2, \ldots, r+c)$ is a set of partial multiplicities $p_{i j}\left(j=1,2, \ldots, g_{i}\right)$ arranged in order of decreasing size: $p_{i 1} \geq p_{i 2} \geq \cdots \geq p_{i g_{i}}$. Instead of (2.30), we now use

$$
J_{F}=\bigoplus_{i=1}^{r+c}\left(\bigoplus_{j=1}^{g_{i}} J_{i j}\right)
$$

to construct a real finite Jordan form. In (3.38), $J_{i j}$ is the familiar $p_{i j} \times p_{i j}$ Jordan block for the real eigenvalues $(i=1,2, \ldots, r)$; for the nonreal eigenvalues $(i=r+1, r+2, \ldots, r+c), J_{i j}$ is a $2 p_{i j} \times 2 p_{i j}$ real Jordan block:

$$
J_{i j}=\left[\begin{array}{ccccc}
C_{i} & I & 0 & \cdots & 0 \\
0 & C_{i} & I & \cdots & 0 \\
\vdots & \vdots & \ddots & \ddots & \vdots \\
0 & 0 & \cdots & C_{i} & I \\
0 & 0 & \cdots & 0 & C_{i}
\end{array}\right], \quad \text { where } C_{i}=\left[\begin{array}{cc}
\sigma_{i} & \omega_{i} \\
-\omega_{i} & \sigma_{i}
\end{array}\right] \in \mathbb{R}^{2 \times 2}
$$

Second, we must update our process for generating the commutant component $S_{F}$ so that it is real and conformable to the revised structure of the real finite Jordan form $J_{F}$ given by (3.38) and (3.39):

$$
S_{F}=\bigoplus_{i=1}^{r+c} S_{i}, \quad \text { where } \quad S_{i}=\left[S_{i, j k}\right] \quad\left(j, k=1,2, \ldots, g_{i}\right),
$$

instead of (2.32). For the real eigenvalues $(i=1,2, \ldots, r)$, we construct the submatrices $S_{i, j k}$ in the same manner as in the case of complex polynomials (see (2.33)-(2.35)), except $S_{i, j k}$ are now real because the free 
Electronic Journal of Linear Algebra, ISSN 1081-3810

A publication of the International Linear Algebra Society

Volume 36, pp. 723-743, November 2020 .

parameters contained therein are taken to be real. For the nonreal eigenvalues $(i=r+1, r+2, \ldots, r+c)$,

$$
\begin{aligned}
& S_{i, j k}=\left[\begin{array}{cccc}
1 A_{i, j k} & { }_{2} A_{i, j k} & \cdots & { }_{p i k} A_{i, j k} \\
0 & { }_{1} A_{i, j k} & \cdots & p_{i k}-1 A_{i, j k} \\
\vdots & \vdots & \ddots & \vdots \\
0 & 0 & \cdots & { }_{1} A_{i, j k} \\
0 & 0 & \cdots & 0 \\
\vdots & \vdots & & \vdots \\
0 & 0 & \cdots & 0
\end{array}\right] \in \mathbb{R}^{2 p_{i j} \times 2 p_{i k}} \quad \text { if } j<k \\
& S_{i, j k}=\left[\begin{array}{cccc}
1 A_{i, j k} & { }_{2} A_{i, j k} & \cdots & { }_{p} A_{i j} A_{i, j} \\
0 & { }_{1} A_{i, j k} & \cdots & p_{i j}-1 A_{i, j k} \\
\vdots & \vdots & \ddots & \vdots \\
0 & 0 & \cdots & { }_{1} A_{i, j k}
\end{array}\right] \in \mathbb{R}^{2 p_{i j} \times 2 p_{i j}} \quad \text { if } j=k \\
& S_{i, j k}=\left[\begin{array}{ccccccc}
0 & \cdots & 0 & { }_{1} A_{i, j k} & { }_{2} A_{i, j k} & \cdots & { }_{p i j} A_{i, j k} \\
0 & \cdots & 0 & 0 & { }_{1} A_{i, j k} & \cdots & p_{i j}-1 A_{i, j k} \\
\vdots & & \vdots & \vdots & \vdots & \ddots & \vdots \\
0 & \cdots & 0 & 0 & 0 & \cdots & { }_{1} A_{i, j k}
\end{array}\right] \in \mathbb{R}^{2 p_{i j} \times 2 p_{i k}} \quad \text { if } j>k,
\end{aligned}
$$

where

$$
{ }_{m} A_{i, j k}=\left[\begin{array}{cc}
{ }_{m} \alpha_{i, j k} & -\left({ }_{m} \beta_{i, j k}\right) \\
{ }_{m} \beta_{i, j k} & { }_{m} \alpha_{i, j k}
\end{array}\right] \in \mathbb{R}^{2 \times 2}
$$

Let us now demonstrate the procedure for constructing a real parameterized structure-preserving transformation with an example.

\subsection{An example. Suppose}

$$
P(\lambda)=A_{3} \lambda^{3}+A_{2} \lambda^{2}+A_{1} \lambda+A_{0}=\left[\begin{array}{ll}
1 & 1 \\
1 & 1
\end{array}\right] \lambda^{3}+\left[\begin{array}{ll}
1 & 0 \\
1 & 0
\end{array}\right] \lambda^{2}+\left[\begin{array}{ll}
1 & 0 \\
1 & 1
\end{array}\right] \lambda+\left[\begin{array}{ll}
0 & 0 \\
1 & 0
\end{array}\right]
$$

The finite eigenvalues of this polynomial are 0 , which forms a $2 \times 2$ Jordan block $\left(g_{1}=1\right.$ and $\left.p_{11}=2\right)$, and $\pm \mathrm{i}$, both of which are simple $\left(g_{i}=1(i=2,3)\right.$ and $\left.p_{i 1}=1\right)$. The infinite eigenvalue also forms a $2 \times 2$ Jordan block $\left(g_{\infty}=1\right.$ and $\left.p_{\infty 1}=2\right)$. Therefore, from (2.31), (3.38), and (3.39), we have that real finite and infinite Jordan forms of $P(\lambda)$ are, respectively,

$$
J_{F}=\left[\begin{array}{ll}
0 & 1 \\
0 & 0
\end{array}\right] \oplus\left[\begin{array}{cc}
0 & 1 \\
-1 & 0
\end{array}\right]=\left[\begin{array}{cccc}
0 & 1 & 0 & 0 \\
0 & 0 & 0 & 0 \\
0 & 0 & 0 & 1 \\
0 & 0 & -1 & 0
\end{array}\right] \quad \text { and } \quad J_{\infty}=\left[\begin{array}{ll}
0 & 1 \\
0 & 0
\end{array}\right]
$$


Isospectral to $P(\lambda)$ is the polynomial

$$
\widetilde{P}(\lambda)=\widetilde{A}_{3} \lambda^{3}+\widetilde{A}_{2} \lambda^{2}+\widetilde{A}_{1} \lambda+\widetilde{A}_{0}=\left[\begin{array}{ll}
0 & 1 \\
0 & 0
\end{array}\right] \lambda^{3}+\left[\begin{array}{cc}
-1 & 0 \\
0 & 0
\end{array}\right] \lambda^{2}+\left[\begin{array}{cc}
0 & 1 \\
-1 & 0
\end{array}\right] \lambda+\left[\begin{array}{ll}
1 & 0 \\
0 & 0
\end{array}\right] .
$$

For this example, we will generate a real parameterized structure-preserving transformation of $P(\lambda)$ and $\widetilde{P}(\lambda)$ using the Fiedler pencil

$$
C_{F_{\sigma}}(\lambda)=M_{3} \lambda-M_{1} M_{0} M_{2}=\left[\begin{array}{ccc}
A_{3} & 0 & 0 \\
0 & I & 0 \\
0 & 0 & I
\end{array}\right] \lambda-\left[\begin{array}{ccc}
-A_{2} & I & 0 \\
-A_{1} & 0 & -A_{0} \\
I & 0 & 0
\end{array}\right],
$$

and so the associated companion forms of $P(\lambda)$ and $\widetilde{P}(\lambda)$ are, respectively,

$$
A \lambda-B=\left[\begin{array}{cccccc}
1 & 1 & 0 & 0 & 0 & 0 \\
1 & 1 & 0 & 0 & 0 & 0 \\
0 & 0 & 1 & 0 & 0 & 0 \\
0 & 0 & 0 & 1 & 0 & 0 \\
0 & 0 & 0 & 0 & 1 & 0 \\
0 & 0 & 0 & 0 & 0 & 1
\end{array}\right] \lambda-\left[\begin{array}{cccccc}
-1 & 0 & 1 & 0 & 0 & 0 \\
-1 & 0 & 0 & 1 & 0 & 0 \\
-1 & 0 & 0 & 0 & 0 & 0 \\
-1 & -1 & 0 & 0 & -1 & 0 \\
1 & 0 & 0 & 0 & 0 & 0 \\
0 & 1 & 0 & 0 & 0 & 0
\end{array}\right]
$$

and

$$
\widetilde{A} \lambda-\widetilde{B}=\left[\begin{array}{cccccc}
0 & 1 & 0 & 0 & 0 & 0 \\
0 & 0 & 0 & 0 & 0 & 0 \\
0 & 0 & 1 & 0 & 0 & 0 \\
0 & 0 & 0 & 1 & 0 & 0 \\
0 & 0 & 0 & 0 & 1 & 0 \\
0 & 0 & 0 & 0 & 0 & 1
\end{array}\right] \lambda-\left[\begin{array}{cccccc}
1 & 0 & 1 & 0 & 0 & 0 \\
0 & 0 & 0 & 1 & 0 & 0 \\
0 & -1 & 0 & 0 & -1 & 0 \\
1 & 0 & 0 & 0 & 0 & 0 \\
1 & 0 & 0 & 0 & 0 & 0 \\
0 & 1 & 0 & 0 & 0 & 0
\end{array}\right] .
$$

In our previous example in Section 2.4, we provided a detailed illustration of the method in Theorem 2.1 and Lemma 2.2 for obtaining a structure-preserving transformation's component matrices. For brevity in this example, we simply state our findings for the strong linearizations (3.45) and (3.46), and we encourage the reader to confirm our results using (2.10):

$$
\begin{aligned}
& Q=\left[\begin{array}{cccccc}
0 & -1 & 1 & 1 & 1 & 0 \\
0 & -1 & 1 & 1 & 1 & -1 \\
0 & 0 & 0 & 1 & 1 & -1 \\
0 & 0 & 0 & 1 & 0 & 0 \\
0 & 1 & 0 & -1 & -1 & 1 \\
-1 & -1 & 1 & 0 & 1 & 0
\end{array}\right], \quad R=\left[\begin{array}{cccccc}
0 & 0 & 1 & 0 & -1 & 1 \\
0 & -1 & 0 & 1 & 1 & 0 \\
0 & 0 & 0 & 1 & 0 & 1 \\
0 & 0 & 0 & 1 & 0 & 0 \\
0 & 1 & 0 & -1 & 0 & -1 \\
-1 & -1 & 1 & 0 & 0 & 1
\end{array}\right], \\
& \widetilde{Q}=\left[\begin{array}{cccccc}
0 & 1 & -1 & 0 & 1 & 0 \\
0 & 0 & 0 & 0 & 0 & 1 \\
0 & 0 & 0 & -1 & 0 & -2 \\
0 & 0 & 0 & 0 & 1 & 0 \\
0 & -1 & 0 & 0 & 1 & 0 \\
1 & 0 & 0 & 1 & 0 & 1
\end{array}\right], \quad \text { and } \widetilde{R}=\left[\begin{array}{cccccc}
0 & 0 & 0 & 0 & 1 & 0 \\
0 & 1 & -1 & 0 & 0 & 1 \\
0 & 0 & 0 & -1 & 0 & 0 \\
0 & 0 & 0 & 0 & 0 & 1 \\
0 & -1 & 0 & 0 & 0 & 1 \\
1 & 0 & 0 & 1 & 0 & 0
\end{array}\right] .
\end{aligned}
$$


Electronic Journal of Linear Algebra, ISSN 1081-3810

A publication of the International Linear Algebra Society

Volume 36, pp. 723-743, November 2020

Now, by (2.20) and (2.21), the dimension of $\Phi$ is 6 , which is consistent with the 6 free parameters that comprise the commutant $S=S_{F} \oplus S_{\infty}$ :

$$
S_{F}=\left[\begin{array}{cc}
a & b \\
0 & a
\end{array}\right] \oplus\left[\begin{array}{cc}
c & -d \\
d & c
\end{array}\right]=\left[\begin{array}{cccc}
a & b & 0 & 0 \\
0 & a & 0 & 0 \\
0 & 0 & c & -d \\
0 & 0 & d & c
\end{array}\right] \quad \text { and } \quad S_{\infty}=\left[\begin{array}{ll}
e & f \\
0 & e
\end{array}\right]
$$

where we used a combination of (2.32)-(2.35) and (3.40)-(3.44). So long as these parameters are real and chosen to make $S$ invertible, the matrices

$$
U=\widetilde{Q} S Q^{-1}=\left[\begin{array}{cccccc}
e+f & -c-e-f & a+e & d-e & a-c & 0 \\
e & -e & 0 & 0 & 0 & 0 \\
-2 e & -d+2 e & 0 & -c & -d & 0 \\
e+f & -e-f & e & -e & 0 & 0 \\
e+f & -e-f & -a+e & -e & -a & 0 \\
a+e & d-e & b & -a+c & b+d & -a
\end{array}\right]
$$

and

$$
V=\widetilde{R} S R^{-1}=\left[\begin{array}{cccccc}
0 & e & e+f & -e-f & e & 0 \\
-c & -c & a+e & d-e & a-c & 0 \\
-d & -d & 0 & -c & -d & 0 \\
0 & 0 & e & -e & 0 & 0 \\
0 & 0 & -a+e & -e & -a & 0 \\
a+d & a+d & b & -a+c & b+d & -a
\end{array}\right]
$$

of Theorem 2.5 define all real structure-preserving transformations that relate the isospectral polynomials $P(\lambda)$ and $\widetilde{P}(\lambda)$ through their companion forms (3.45) and (3.46), respectively.

4. Diagonalizable quadratic matrix polynomials. We now discuss the special case of isospectral matrix polynomials $P(\lambda)$ and $\widetilde{P}(\lambda)$ that have degree two and where $\widetilde{P}(\lambda)$ is diagonal; we say $P(\lambda)$ is diagonalizable in this case. With regard to applications, the issue of diagonalizability arises in the analysis of small-amplitude oscillatory behavior (e.g., see [14]). A typical approach involves transforming the governing system of linear second-order differential equations into a set of mutually independent equations for further analysis. These systems of equations are intimately related to isospectral quadratic polynomials, so the transformation involved is one that converts a quadratic polynomial into diagonal form while preserving its finite and infinite Jordan structures. In fact, we considered this very topic in an earlier work [9] for real quadratic polynomials, which we later discuss in Section 4.2.

4.1. Complex matrix polynomials. When the leading coefficient of a complex quadratic polynomial $P(\lambda)$ is singular, Zúñiga Anaya demonstrated in [16] that a diagonal quadratic polynomial $\widetilde{P}(\lambda)$ isospectral to $P(\lambda)$ exists if and only if the following conditions hold (see Theorem 2 in [16] and Zúñiga Anaya's discussion of its proof):

(i) the Jordan blocks of all eigenvalues (finite and infinite) must be no larger than $2 \times 2$; and

(ii) excluding the $2 \times 2$ Jordan blocks, all remaining eigenvalues, which necessarily have unit partial multiplicities, must form pairs of differing eigenvalues. 
These restrictions on the shared finite and infinite Jordan structures of $P(\lambda)$ and $\widetilde{P}(\lambda)$ mean that the eigenvalues form pairs in such a way that the diagonal of $\widetilde{P}(\lambda)$ is populated by three types of terms [16]:

(i) A quadratic term formed by a pairing of two finite eigenvalues: $\left(\lambda-\lambda_{j}\right)\left(\lambda-\lambda_{k}\right)=\lambda^{2}-\left(\lambda_{j}+\lambda_{k}\right) \lambda+$ $\lambda_{j} \lambda_{k}$, where $\lambda_{j}$ is the eigenvalue in the $j$ th row and column of the finite Jordan form $J_{F}$, and similarly for $\lambda_{k}$. This pair of finite eigenvalues $\left(\lambda_{j}, \lambda_{k}\right)$ can consist of different eigenvalues with unit partial multiplicities $\left(\lambda_{j} \neq \lambda_{k}\right)$ or identical eigenvalues associated with a $2 \times 2$ Jordan block (i.e., $\left.\lambda_{j}=\lambda_{k}=\lambda_{j+1}\right)$.

(ii) A linear term, $\lambda-\lambda_{j}$, resulting from pairing a finite eigenvalue $\lambda_{j}$ with an eigenvalue at infinity that corresponds to the zero eigenvalue in the $k$ th row and column of the infinite Jordan form $J_{\infty}$ : $\left(\lambda_{j}, \infty_{k}\right)$. Both of these eigenvalues have unit partial multiplicity.

(iii) A constant entry of 1 that arises from pairing a defective infinite eigenvalue with itself: $\left(\infty_{j}, \infty_{j+1}\right)$. These infinite eigenvalues are associated with the $2 \times 2$ zero-eigenvalue Jordan block occupying rows and columns $j$ and $j+1$ of $J_{\infty}$.

In general, the pairing of eigenvalues for a diagonalizable polynomial $P(\lambda)$ is not unique. Moreover, the order in which the quadratic, linear, and constant terms appear on the diagonal of $\widetilde{P}(\lambda)$ is arbitrary. While $\widetilde{P}(\lambda)$ might not be unique, all possible forms are members of an equivalence class because $P(\lambda)$ and $\widetilde{P}(\lambda)$ are isospectral.

4.1.1. The first companion form. Recall from Section 2.2 .1 that when our strong linearization of choice is the first companion form (2.6), the matrices $Q$ and $R$ that comprise the corresponding structurepreserving transformation can be formed from the coefficients and spectral data of a polynomial $P(\lambda)$ and its reversal $P_{\text {rev }}(\lambda)$; the same is true of the matrices $\widetilde{Q}$ and $\widetilde{R}$ for a polynomial $\widetilde{P}(\lambda)$ isospectral to $P(\lambda)$. Featured in $\widetilde{Q}$ and $\widetilde{R}$ are the matrices $\widetilde{V}_{F}$ and $\widetilde{V}_{\infty}$ that contain, respectively, the Jordan chains associated with the finite eigenvalues of $\widetilde{P}(\lambda)$ and the zero eigenvalue of the reverse polynomial $\widetilde{P}_{\text {rev }}(\lambda)$ (see Section 2.2.1). Let $e_{i}(i=1,2, \ldots, n)$ be an $n$-dimensional vector of zeros except for a 1 in the $i$ th row. When $\widetilde{P}(\lambda)$ is quadratic and diagonal, all of its eigenvalues form Jordan blocks no larger than $2 \times 2$, and so it is straightforward to show that we can always take $e_{i}$ as eigenvectors and 0 as generalized eigenvectors. Consequently, $\widetilde{V}_{F}$ and $\widetilde{V}_{\infty}$ have very simple forms in this case, and we build these matrices as follows. Suppose $\widetilde{\rho}_{i}(\lambda)$ is the scalar polynomial in the $i$ th row and column of $\widetilde{P}(\lambda)$ :

(i) If $\widetilde{\rho}_{i}(\lambda)=\left(\lambda-\lambda_{j}\right)\left(\lambda-\lambda_{k}\right)$, then place $e_{i}$ in the $j$ th and $k$ th columns of $\widetilde{V}_{F}$ when $\lambda_{j} \neq \lambda_{k}$, or place $e_{i}$ in the $j$ th column and 0 in column $k=j+1$ when $\lambda_{j}=\lambda_{k}$.

(ii) If $\widetilde{\rho}_{i}(\lambda)=\lambda-\lambda_{j}$, then place $e_{i}$ in the $j$ th column of $\widetilde{V}_{F}$ and in the $k$ th column of $\widetilde{V}_{\infty}$.

(iii) If $\widetilde{\rho}_{i}(\lambda)=1$, then make the $i$ th row of $\widetilde{V}_{F}$ a row of zeros, and place $e_{i}$ in the $j$ th column of $\widetilde{V}_{\infty}$ and 0 in column $j+1$.

4.2. Real matrix polynomials. If a real quadratic polynomial $P(\lambda)$ is diagonalizable, then the spectrum of $P(\lambda)$ is subject to an additional constraint: the conjugate pairs of nonreal eigenvalues must be semisimple [12, Theorem 7]. Consequently, nonreal eigenvalues are associated only with quadratic terms in a real diagonal polynomial $\widetilde{P}(\lambda)$ isospectral to $P(\lambda)$, and the only $2 \times 2$ Jordan blocks allowed are those formed by real eigenvalues and the eigenvalue at infinity [9, Corollary 3.3$]$.

4.2.1. The first companion form. Suppose we choose to construct a real parameterized structurepreserving transformation that diagonalizes a real quadratic $P(\lambda)$ through its first companion form; this transformation utilizes the Jordan chains associated with $P(\lambda), \widetilde{P}(\lambda)$, and their reversals. The Jordan 
Electronic Journal of Linear Algebra, ISSN 1081-3810

A publication of the International Linear Algebra Society

Volume 36, pp. 723-743, November 2020 .

chains for the finite eigenvalues of $P(\lambda)$ are still computed according to (2.12), but we form the matrix $V_{F}$ using only real vectors. The eigenvectors and generalized eigenvectors for a real finite eigenvalue can always be taken to be real when $P(\lambda)$ is real. Because the nonreal finite eigenvalues occur in conjugate pairs, so do their corresponding eigenvectors and generalized eigenvectors. If $v_{i} \in \mathbb{C}^{n}$ is an eigenvector (or generalized eigenvector) associated with a nonreal eigenvalue $\lambda_{i}$, then we replace $v_{i}$ and its conjugate $\bar{v}_{i}$ with the real and imaginary parts of $v_{i}$, respectively. The infinite Jordan form $J_{\infty}$ is always real, so the associated Jordan chains in $V_{\infty}$ can also be chosen to be real vectors. The process described in Section 4.1.1 for building the matrices $\widetilde{V}_{F}$ and $\widetilde{V}_{\infty}$ remains valid.

4.3. An example. Consider the quadratic matrix polynomial

$$
P(\lambda)=A_{2} \lambda^{2}+A_{1} \lambda+A_{0}=\left[\begin{array}{cc}
0 & 0 \\
0 & 1 / 2
\end{array}\right] \lambda^{2}+\left[\begin{array}{cc}
1 & 0 \\
1 & 1
\end{array}\right] \lambda+\left[\begin{array}{cc}
1 & -1 \\
1 & 0
\end{array}\right]
$$

The finite eigenvalues of $P(\lambda)$ are -1 and $-1 \pm \mathrm{i}$, all of which are simple $\left(g_{i}=1(i=1,2,3)\right.$ and $\left.p_{i 1}=1\right)$, and thus, the eigenvalue at infinity occurs only once $\left(g_{\infty}=1\right.$ and $\left.p_{\infty 1}=1\right)$. Choosing to represent the Jordan matrix of the finite eigenvalues in real form, from (2.31), (3.38), and (3.39), we have

$$
J_{F}=-1 \oplus\left[\begin{array}{cc}
-1 & 1 \\
-1 & -1
\end{array}\right]=\left[\begin{array}{ccc}
-1 & 0 & 0 \\
0 & -1 & 1 \\
0 & -1 & -1
\end{array}\right] \text { and } \quad J_{\infty}=0
$$

as real finite and infinite Jordan forms of $P(\lambda)$, respectively. We can diagonalize $P(\lambda)$ because its eigenvalues form the admissible pairs $(-1+\mathrm{i},-1-\mathrm{i})$ and $(-1, \infty)$, in which case a diagonal polynomial isospectral to $P(\lambda)$ is

$$
\widetilde{P}(\lambda)=\left[\begin{array}{cc}
\lambda^{2}+2 \lambda+2 & 0 \\
0 & \lambda+1
\end{array}\right]=\left[\begin{array}{ll}
1 & 0 \\
0 & 0
\end{array}\right] \lambda^{2}+\left[\begin{array}{ll}
2 & 0 \\
0 & 1
\end{array}\right] \lambda+\left[\begin{array}{ll}
2 & 0 \\
0 & 1
\end{array}\right]=\widetilde{A}_{2} \lambda^{2}+\widetilde{A}_{1} \lambda+\widetilde{A}_{0}
$$

This polynomial is actually unique up to scaling and permutation of its diagonal entries because $(-1+\mathrm{i},-1-\mathrm{i})$ and $(-1, \infty)$ are the only allowable eigenvalue pairs. In this example, we will construct a real parameterized structure-preserving transformation of $P(\lambda)$ and $\widetilde{P}(\lambda)$ through their first companion forms:

$$
A \lambda-B=\left[\begin{array}{cc}
A_{2} & 0 \\
0 & I
\end{array}\right] \lambda-\left[\begin{array}{cc}
-A_{1} & -A_{0} \\
I & 0
\end{array}\right]=\left[\begin{array}{cccc}
0 & 0 & 0 & 0 \\
0 & 1 / 2 & 0 & 0 \\
0 & 0 & 1 & 0 \\
0 & 0 & 0 & 1
\end{array}\right] \lambda-\left[\begin{array}{cccc}
-1 & 0 & -1 & 1 \\
-1 & -1 & -1 & 0 \\
1 & 0 & 0 & 0 \\
0 & 1 & 0 & 0
\end{array}\right]
$$

and

$$
\widetilde{A} \lambda-\widetilde{B}=\left[\begin{array}{cccc}
1 & 0 & 0 & 0 \\
0 & 0 & 0 & 0 \\
0 & 0 & 1 & 0 \\
0 & 0 & 0 & 1
\end{array}\right] \lambda-\left[\begin{array}{cccc}
-2 & 0 & -2 & 0 \\
0 & -1 & 0 & -1 \\
1 & 0 & 0 & 0 \\
0 & 1 & 0 & 0
\end{array}\right]
$$

respectively, from (2.6). Consequently, we will need the Jordan chains associated with $J_{F}$ and $J_{\infty}$ for both $P(\lambda)$ and $\widetilde{P}(\lambda)$. For $P(\lambda)$, we follow Section 2.2.1 and find from $(2.12)$ that $[1,0]^{T}$ and $[1, \pm \mathrm{i}]^{T}$ are eigenvectors that correspond to the finite eigenvalues -1 and $-1 \pm \mathrm{i}$, respectively, while $[1,0]^{T}$ is an eigenvector for the 
infinite eigenvalue according to (2.13). Therefore, in real form,

$$
V_{F}=\left[\begin{array}{lll}
1 & 1 & 0 \\
0 & 0 & 1
\end{array}\right] \quad \text { and } \quad V_{\infty}=\left[\begin{array}{l}
1 \\
0
\end{array}\right]
$$

For $\widetilde{P}(\lambda)$, we have from Section 4.1.1 that

$$
\widetilde{V}_{F}=\left[\begin{array}{lll}
0 & 1 & 1 \\
1 & 0 & 0
\end{array}\right] \quad \text { and } \quad \widetilde{V}_{\infty}=\left[\begin{array}{l}
0 \\
1
\end{array}\right]
$$

As a result, by (2.14), the transformation matrices

$$
\begin{aligned}
Q & =\left[\begin{array}{cccc}
0 & 0 & 0 & -1 \\
0 & -1 / 2 & -1 / 2 & -1 \\
1 & 1 & 0 & 1 \\
0 & 0 & 1 & 0
\end{array}\right], \quad R=\left[\begin{array}{cccc}
-1 & -1 & 1 & 1 \\
0 & -1 & -1 & 0 \\
1 & 1 & 0 & 0 \\
0 & 0 & 1 & 0
\end{array}\right], \\
\widetilde{Q} & =\left[\begin{array}{cccc}
0 & -2 & 0 & 0 \\
0 & 0 & 0 & -1 \\
0 & 1 & 1 & 0 \\
1 & 0 & 0 & 1
\end{array}\right], \quad \text { and } \quad \widetilde{R}=\left[\begin{array}{cccc}
0 & -2 & 0 & 0 \\
-1 & 0 & 0 & 1 \\
0 & 1 & 1 & 0 \\
1 & 0 & 0 & 0
\end{array}\right] .
\end{aligned}
$$

Now, evaluating (2.20) and (2.21) reveals that the dimension of $\Phi$ is 4 , which we observe from the 4 free parameters present in the commutant $S=S_{F} \oplus S_{\infty}$ that we obtain from a combination of (2.32)-(2.35) and (3.40)-(3.44):

$$
S_{F}=a \oplus\left[\begin{array}{cc}
b & -c \\
c & b
\end{array}\right]=\left[\begin{array}{ccc}
a & 0 & 0 \\
0 & b & -c \\
0 & c & b
\end{array}\right] \quad \text { and } \quad S_{\infty}=d
$$

Thus, if we limit these 4 parameters to real values that make $S$ nonsingular, then we have from Theorem 2.5 that all real structure-preserving transformations $(U, V)$ connecting $P(\lambda)$ and its diagonal form $\widetilde{P}(\lambda)$ through their respective first companion forms (4.47) and (4.48) are generated from

$$
U=\widetilde{Q} S Q^{-1}=\left[\begin{array}{cccc}
-4 b & 4 b & 0 & 2 b+2 c \\
d & 0 & 0 & 0 \\
2 b+2 c & -2 b-2 c & 0 & -2 c \\
-a-d & 2 a & a & a
\end{array}\right]
$$

and

$$
V=\widetilde{R} S R^{-1}=\left[\begin{array}{cccc}
0 & 2 b & 0 & 2 b+2 c \\
d & -a & -a+d & -a-d \\
0 & -b-c & 0 & -2 c \\
0 & a & a & a
\end{array}\right] .
$$

5. Closing remarks. We have shown that the strict equivalence transformation relating the companion forms of two regular and isospectral matrix polynomials can be parameterized by a matrix that commutes 
with the companion forms' shared Weierstrass canonical form; this commutant must be a block-diagonal matrix $S_{F} \oplus S_{\infty}$ whose blocks $S_{F}$ and $S_{\infty}$ separately commute with the polynomials' common finite and infinite Jordan forms, respectively. Our result is a direct generalization of prior work by Lancaster and Zaballa [11] for the case when the polynomials' leading coefficients are invertible, and it holds for any choice of companion form. We demonstrated this generality through several examples that each utilized a different Fiedler pencil, one being the familiar first companion form. Moreover, we illustrated how to accommodate diagonalizable quadratic polynomials that arise in various applications.

Acknowledgments. We are grateful to an anonymous reviewer for providing constructive feedback that helped to improve this paper.

\section{REFERENCES}

[1] E.N. Antoniou and S. Vologiannidis. A new family of companion forms of polynomial matrices. Electron. J. Linear Algebra, 11:78-87, 2004.

[2] F. De Terán, F.M. Dopico, and D.S. Mackey. Fiedler companion linearizations for rectangular matrix polynomials. Linear Algebra Appl., 437:957-991, 2012.

[3] F. De Terán, F.M. Dopico, and D.S. Mackey. Fiedler companion linearizations and the recovery of minimal indices. SIAM J. Matrix Anal. Appl., 31:2181-2204, 2010.

[4] M. Fiedler. A note on companion matrices. Linear Algebra Appl., 372:325-331, 2003.

[5] F.R. Gantmacher. The Theory of Matrices. Chelsea Publishing Company, New York, 1959.

[6] I. Gohberg, M.A. Kaashoek, and P. Lancaster. General theory of regular matrix polynomials and band Toeplitz operators. Integral Equations Operator Theory, 11:776-882, 1988.

[7] I. Gohberg, P. Lancaster, and L. Rodman. Invariant Subspaces of Matrices with Applications. John Wiley \& Sons, Inc., New York, 1986.

[8] I. Gohberg, P. Lancaster, and L. Rodman. Matrix Polynomials. Academic Press, New York, 1982.

[9] D.T. Kawano, R.G. Salsa, and F. Ma. Decoupling of second-order linear systems by isospectral transformation. Z. Angew. Math. Phys., 69:137, 2018.

[10] P. Lancaster and P. Psarrakos. A note on weak and strong linearizations of regular matrix polynomials. Numerical Analysis Report 470, Manchester Centre for Computational Mathematics, Manchester, 2005.

[11] P. Lancaster and I. Zaballa. Parametrizing structure preserving transformations of matrix polynomials. Oper. Theory Adv. Appl., 218:403-424, 2012.

[12] P. Lancaster and I. Zaballa. Diagonalizable quadratic eigenvalue problems. Mech. Syst. Signal Process., 23:1134-1144, 2009.

[13] D.S. Mackey, N. Mackey, C. Mehl, and V. Mehrmann. Vector spaces of linearizations for matrix polynomials. SIAM J. Matrix Anal. Appl., 28:971-1004, 2006.

[14] L. Meirovitch. Principles and Techniques of Vibrations. Prentice Hall, Upper Saddle River, 1997.

[15] S. Vologiannidis and E.N. Antoniou. A permuted factors approach for the linearization of polynomial matrices. Math. Control Signals Systems, 22:317-342, 2011.

[16] J.C. Zúñiga Anaya. Diagonalization of quadratic matrix polynomials. Systems Control Lett., 59:105-113, 2010. 